\title{
Fabrication of Tailorable pH Responsive Cationic Amphiphilic Microgels on a Microfluidic Device for Drug Release
}

\author{
Bingyuan Lu, ${ }^{1}$ Mark D. Tarn, ${ }^{1}$ Nicole Pamme,${ }^{1}$ Theoni K. Georgiou ${ }^{2}$ \\ ${ }^{1}$ School of Mathematics and Physical Sciences, University of Hull, Hull, United Kingdom, HU6 7RX \\ 2Department of Materials, Imperial College London, Royal School of Mines, Exhibition Road, London, \\ United Kingdom, SW7 2AZ \\ Correspondence to: Nicole Pamme (E-mail: n.pamme@hull.ac.uk); Theoni K. Georgiou (Email: \\ t.georgiou@imperial.ac.uk)
}

\begin{abstract}
Cationic, amphiphilic microgels of differing compositions based on hydrophilic, $\mathrm{pH}$ - and thermoresponsive 2-(dimethylamino)ethyl methacrylate (DMAEMA) and hydrophobic, non-ionic n-butyl acrylate $(\mathrm{BuA})$ are synthesized using a lab-on-a-chip device. Hydrophobic oil-in-water (o/w) droplets are generated via a microfluidic platform, with the dispersed (droplet) phase containing the DMAEMA and $\mathrm{BuA}$, alongside the hydrophobic cross-linker, ethylene glycol dimethacrylate (EGDMA), and a free radical initiator in an organic solvent. Finally, the hydrophobic droplets are photopolymerized via a UV light source as they traverse the microfluidic channel to produce the cationic amphiphilic microgels. This platform enables the rapid, automated, and in-situ production of amphiphilic microgels, which does not match the core-shell structure of conventionally prepared microgels but are instead based on random amphiphilic copolymers of DMAEMA and BuA between the hydrophobic cross-links. The microgels are characterized in terms of their swelling and encapsulation abilities, which are found to be influenced by both the $\mathrm{pH}$ response and the hydrophobic content of the microgels.
\end{abstract}

KEYWORDS: microgel; microfluidics; photopolymerization; amphiphilic gels; cationic

\section{INTRODUCTION}

Microgels are three-dimensionally cross-linked polymer particles with sizes ranging from 0.1 to $100 \mu \mathrm{m}$, and which have many interesting applications such as drug and gene delivery, ${ }^{1-16}$ tissue engineering, ${ }^{2,17-19}$ biosensing, ${ }^{20,21}$ use in the oil industry, ${ }^{22}$ organic dye removal, ${ }^{23,24}$ coatings, ${ }^{25-27}$ textiles $^{28-30}$ and in the food industry. ${ }^{31}$ Microgels are typically either hydrophilic and are dispersed in water or aqueous media, especially when used for biomedical applications, or they are hydrophobic and thus dispersed in organic solvents in which case they are called latex particles and can be used to prepare materials such as hydrophobic films. Amphiphilic microgels, however, contain both hydrophobic and hydrophilic groups and thus have many intriguing potential applications since they can be dispersed in both aqueous and nonaqueous media. In particular, they are able to encapsulate and deliver both hydrophobic and 
hydrophilic moieties and, like their macrogel ${ }^{32}$ counterparts, they can potentially have better mechanical properties compared to hydrophilic microgels. A drawback to amphiphilic microgels is that they are difficult to manufacture. Most methodologies for microgel preparation involve emulsion polymerization in which droplets containing pre-gels or polymerizable reagents are polymerized to form the microgel. Thus, hydrophilic and hydrophobic microgels are created by polymerizing either water or oil droplets, where the continuous phase $(\mathrm{CP})$ is oil or water, respectively. If a co-monomer of different hydrophilicity/hydrophobicity is incorporated into the droplet, with the aim to produce microgels with both hydrophilic and hydrophobic moieties, the co-monomer will diffuse into the $\mathrm{CP}$ with which it is more compatible. Therefore, the synthesis of amphiphilic microgels is challenging and usually

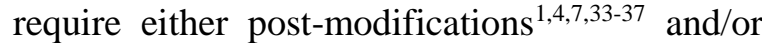
multi-step procedures. ${ }^{6,38} \mathrm{~A}$ common strategy is the fabrication of hydrophilic microgels followed by a modification by covalently or electrostatically binding amphiphilic or hydrophobic moieties. . $^{1,4,734-37}$ However, this results in the synthesis of microgels with a coreshell structure, where the hydrophobic groups are grafted onto the shell of the microgel structure. We are aiming for the fabrication of amphiphilic microgels with different structures, in particular where the hydrophobic and hydrophilic groups are both on the elastic chain, the polymer chain between the cross-links, and not grafted on the outer surface of the microgel that can influence the microgel' $\mathrm{s}$ ability to encapsulate and release drugs. We have previously demonstrated that this structure can be achieved using microfluidic devices. ${ }^{46,47}$

The application of microfluidic devices ${ }^{39}$ for the production of microgels brings with it advantages that include: (i) the formation of droplets, ${ }^{40,41}$ and consequently microgels, of narrower size distribution and (ii) tailoring of the size of the droplets/microgels by varying the applied flow rates of the droplet dispersed phase (DP) and the CP. ${ }^{31,42-45}$ Importantly, they can also be used as platforms to perform rapid reactions ${ }^{46-48}$ and enable the polymerization of unstable and/or easily hydrolysed reagents in flow within the microchannel. In particular, reagents such as (meth)acrylic monomers tend to hydrolyse to (meth)acrylic acid when in contact with water ${ }^{49}$ and thus they cannot be readily dispersed and polymerized in water to produce amphiphilic microgels.

In our previous studies, we have prepared anionic amphiphilic microgels and we investigated how the hydrophobic/hydrophilic ratio and the cross-linking density can affect their swelling and ability to deliver both hydrophobic and hydrophilic payloads. ${ }^{50,51}$ In the present study, we aimed to fabricate cationic microgels, specifically containing hydrophilic 2(dimethylamino)ethyl methacrylate (DMAEMA), as DMAEMA-based polymers are $\mathrm{pH}$ and thermoresponsive and have been used in drug and gene delivery. ${ }^{11-16}$ DMAEMA containing microgels have been synthesized previously using conventional methodologies, but none were amphiphilic or were prepared on a lab-on-a-chip. ${ }^{21,52-64}$ In several studies, DMAEMA was combined with $N$ isopropylacrylamide (NIPAM) to produce dual thermoresponsive microgels, ${ }^{21,52-54,56,64}$ or with other hydrophilic monomers or polymers. ${ }^{57,58,60}$ Thus, to the best of our knowledge, this is the first time that amphiphilic, cationic, DMAEMA containing microgels have been fabricated, made possible via the use of a microfluidic platform.

In this study, the hydrophilic, cationic DMAEMA monomer and hydrophobic $n$-butyl acrylate $(\mathrm{BuA})$ monomer were introduced onto the elastic chain of the microfluidicallyfabricated polymer microgels during production by generation of pre-cursor droplets and subsequent photopolymerization within the microchannel. The ratio of the two monomers was systematically varied without changing the cross-linking density i.e. the amount of the hydrophobic cross-linker ethylene glycol dimethacrylate (EGDMA) to the total amount of the two monomers. The $\mathrm{pH}$ responsiveness of the DMAEMA-based microgels was evaluated by studying their swelling in different aqueous $\mathrm{pH}$ solutions. Finally, to demonstrate the amphiphilic nature of the microgels and their potential for drug delivery, the encapsulation and release of both hydrophobic and hydrophilic moieties was investigated using Nile Red and Eriglaucine, respectively, as model drugs. 


\section{EXPERIMENTAL}

\section{Materials and Methods}

DMAEMA (99 \%), BuA (99 \%), EGDMA (cross-linker), sodium dodecyl sulfate (SDS), 2,2-diphenyl-1-picrylhydrazyl hydrate (DPPH, free radical inhibitor, $99 \%)$, 1-hydroxycyclohexyl phenyl ketone (HCPK, $99 \%$, free radical initiator), tetramethylethylenediamine (TEMED, $99 \%$, free radical accelerator), Nile Red, Eriglaucine disodium salt, phenothiazine (98\%, free radical inhibitor), sulfuric acid, sodium bicarbonate $\left(\mathrm{NaHCO}_{3}\right)$, sodium sulphate $\left(\mathrm{Na}_{2} \mathrm{SO}_{4}\right)$, hydrochloric acid, sodium hydroxide and basic alumina were purchased from SigmaAldrich (Dorset, UK). DMAEMA, BuA and EGDMA were passed through a basic alumina column to remove the free radical initiator prior to use. Chloroform (99\%) and ethanol were purchased from Fisher Scientific (Loughborough, UK). The chloroform was used to prepare the hydrophobic droplets while the ethanol was used for dissolving Nile Red dye. The chemical structures of the main reagents used for microgel fabrication (DMAEMA, BuA and EGDMA) are shown in Figure 1.

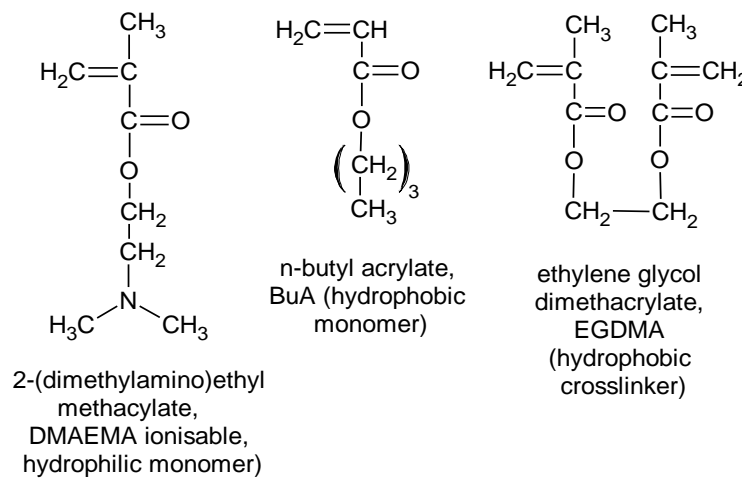

Figure 1 Chemical structures of the monomers (hydrophilic DMAEMA and hydrophobic BuA) and cross-linker (EGDMA).

\section{Microfluidic Chip Fabrication and Setup}

The microfluidic chip used for droplet formation and polymerization consisted of three distinct regions: (i) a flow focusing section for the generation of droplets containing DMAEMA, $\mathrm{BuA}$, and EGDMA, (ii) a short, shallow serpentine mixing channel that allowed rapid mixing of the droplet components, and (iii) a long, wide and deep serpentine channel for UV irradiation of the droplets (Figure 2a). The chip was composed of a top layer and a bottom layer, ${ }^{50,51}$ and was fabricated from glass (B270 glass, Telic, CA, USA) using conventional photolithography and wet etching techniques. ${ }^{65}$ The design in the top layer was etched to a depth of $10 \mu \mathrm{m}$ and featured the flow focusing droplet generation section and a short serpentine mixing section with channel widths of $30 \mu \mathrm{m}$, as well as a long, wide serpentine channel that had a channel width of $720 \mu \mathrm{m}$ and a length of $188.35 \mathrm{~cm}$. The bottom layer featured only the long, wide serpentine channel, as a mirror image to the complementary design on the top later, that was etched to a depth of $50 \mu \mathrm{m}$ with a width of 800 $\mu \mathrm{m}$. The two layers were aligned and thermally bonded in a furnace at $585{ }^{\circ} \mathrm{C}$ for $3 \mathrm{~h}$, yielding a final depth for the long and wide serpentine channel of $60 \mu \mathrm{m}$ (Figure 2b).

Fused silica capillaries $(150 \mu \mathrm{m}$ i.d., $363 \mu \mathrm{m}$ o.d., CM Scientific, UK) were glued into the inlet and outlet holes and connected to $500 \mu \mathrm{L}$ glass syringes (SGE, Sigma-Aldrich, UK) via syringe adaptors (Kinesis, UK) (Figure 2c). Two syringe pumps (PHD2000, Harvard Apparatus, UK) were used to control the flow rates of the $\mathrm{CP}$ and DP between $0.5 \mu \mathrm{L} \mathrm{min}{ }^{-1}$ and $5 \mu \mathrm{L} \mathrm{min}^{-1}$. Aluminium foil was used to cover the syringes and the droplet generation section on the chip in order to prevent the reagents from polymerizing prior to droplet formation. Photopolymerization in the long serpentine channel was achieved using a $12 \mathrm{~W}$, $365 \mathrm{~nm}$ UV light source (XX-15S, Ultra-Violet Products Ltd., UK). The entire setup was covered with a thick black cloth in order to protect users from UV light. The microgels were collected in a glass vial.

Videos and images of on-chip droplet generation were obtained using a colour CCD camera (MTV-63V1N, Mintron, Taiwan) attached to an inverted microscope (Eclipse Ti, Nikon, UK), with images captured using WinDVD Creator 2 (Corel Ltd., UK) software. Collected microgels were observed using either the same microscope setup, or using a second setup consisting of an upright microscope (BH-2, Olympus, UK), a CCD camera (INFINITYlite, Lumenera) and capture software (Studio Capture, 
Mettler-Toledo, Inc.). ImageJ freeware was used for the analysis of droplet size and colour intensity.

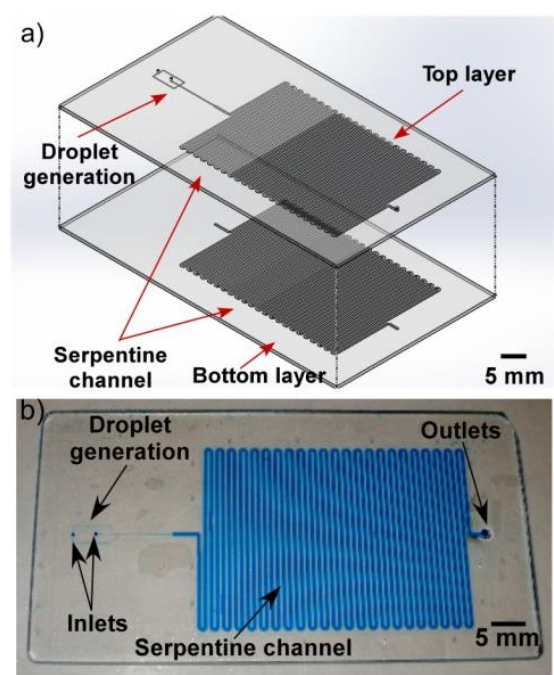

c)

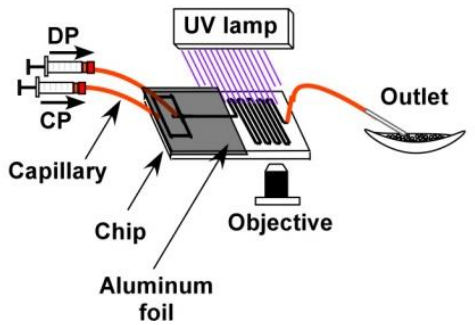

Figure 2 (a) Exploded schematic of the microfluidic device, featuring a flow focusing junction and a long serpentine channel in the top layer (10 $\mu \mathrm{m}$ deep, $30 \mu \mathrm{m}$ wide), and the serpentine channel $(50 \mu \mathrm{m}$ deep, $800 \mu \mathrm{m}$ wide, $188.35 \mathrm{~cm}$ long) mirrored in the bottom layer. (b) Photograph of the microfluidic chip fabricated in glass, with blue dye used to visualise the microchannels. (c) Schematic showing the principle of droplet generation via in situ UV polymerization to form microgels.

\section{Formation of Droplets and Polymerization of Microgels}

The DP was based on chloroform containing DMAEMA, BuA and EGDMA with varying molar ratios of DMAEMA and BuA (though the total concentration of the reagents was always 30 wt $\%$ and thus the monomer cross-linker ratio was always 70:4 DMAEMA/BuA:EGDMA), and 4 wt $\%$ of HCPK (free radical photoinitiator). The $\mathrm{CP}$ was an aqueous solution of $0.1 \mathrm{wt} \% \mathrm{SDS}$ and 2 wt $\%$ TEMED, with the concentration of SDS being below the critical micelle concentration. ${ }^{66}$ During droplet generation optimisation studies, the flow rate of the $\mathrm{CP}$ was varied from $0.5 \mu \mathrm{L}$ $\min ^{-1}$ to $5 \mu \mathrm{L} \mathrm{min}{ }^{-1}$, while the DP flow rate was held at $0.5 \mu \mathrm{L} \mathrm{m^{-1 }}$. Later, microgel fabrication was performed at the $\mathrm{CP}$ and $\mathrm{DP}$ flow rates of 2

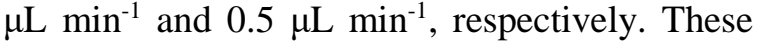
flow rates allowed the droplets to be UV irradiated for $30 \mathrm{~min}$ as they passed through the long wide serpentine channel, forming the microgels. One DMAEMA-EGDMA microgel and three DMAEMA-BuA-EGDMA microgels of differing compositions were formed, with molar ratios of 75:0 49:21, 35:35, and 21:49 DMAEMA:BuA, respectively.

\section{Swelling Studies in Different pHs}

Aqueous solutions of $\mathrm{NaOH}(1 \mathrm{M})$ and $\mathrm{HCl}(1 \mathrm{M})$ were used to vary the $\mathrm{pH}$ of the solution that the DMAEMA-BuA-EGDMA microgels were suspended in, from $\mathrm{pH} 1$ to $\mathrm{pH} 14$. These tests were performed in order to study the responsiveness of the microgel particles. The microgels were photographed using a colour CCD camera and microscope setups described previously, with ImageJ used for image analysis. The extent of swelling and shrinking (relative size) was calculated by dividing the microgel size at a given $\mathrm{pH}$ by their initial size measured at $\mathrm{pH}$ 7.

\section{$\mathrm{pK}_{\mathrm{a}}$ Determination}

The $p \mathrm{~K}_{\mathrm{a}}$ values of the three synthesized types of DMAEMA-BuA-EGDMA microgels were determined by potentiometric titration. Specifically, $0.04 \mathrm{~g}$ of each microgel was dispersed in water and $1 \mathrm{M} \mathrm{HCl}$ added to reduce the $\mathrm{pH}$ to around $\mathrm{pH}$ 2. The titration was performed by adding $0.05 \mathrm{~mL}$ aliquots of $0.05 \mathrm{M}$ $\mathrm{NaOH}$ and measuring the $\mathrm{pH}$ after each addition.

\section{Dye Encapsulation and Release Studies}

Two types of dye, acting as model drugs, were introduced into the microgels via the methodology described below in order to determine the capability of the microgels to encapsulate and release both hydrophilic and hydrophobic moieties. 5 wt $\%$ hydrophobic Nile 
Red in an ethanol/water (1:1 volume ratio) mixture or hydrophilic Eriglaucine in water were loaded into the already polymerized DMAEMAbased microgels in a $\mathrm{pH} 3$ solution by diffusion with sonication for $30 \mathrm{~min}$, before removing the microgels by pipette, adding them to a microscope slide and removing any excess solution. The chemical structures of both dyes are shown in Figure 3. The model drug release was monitored via the change in microgel colour intensity over time, using the colour CCD camera on the microscope. Images were taken at regular time intervals (every $5 \mathrm{~min}$ ) and ImageJ freeware was used for the analysis of colour intensity by converting photographs into 8-bit and measuring the mean grey values inside of the microgels. a)

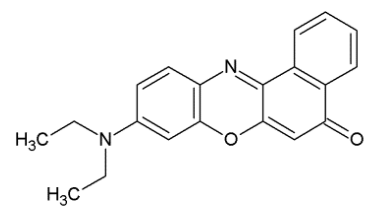

b)

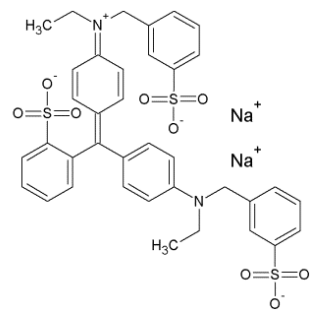

Figure 3 Chemical structures of (a) hydrophobic Nile Red, and (b) hydrophilic Eriglaucine disodium salt.

\section{RESULTS AND DISCUSSION}

\section{Microgel Fabrication and pH Responsiveness Studies}

The microgels were fabricated using the specially developed microfluidic chip employed in our previous study on anionic AA-BuA-EGDMA microgels. ${ }^{50,51} \mathrm{By}$ introducing the $\mathrm{CP}$ and $\mathrm{DP}$ at

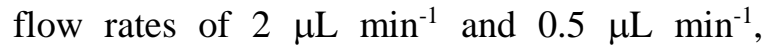
respectively, reagent precursor droplets containing DMAEMA, BuA, EGDMA and $\mathrm{HCPK}$ in chloroform were generated in at the flow focusing junction of the microfluidic device with volumes of $3.1 \mathrm{pL}$ (CV $7 \%$ ). Polymerization of the droplets was performed in situ on the chip via their exposure to UV light as they passed through the large serpentine channel. This was crucial since the hydrophilic DMAEMA monomer is easily hydrolysable in water, ${ }^{49}$ but by immediately subjecting the droplets to UV they could be polymerized without the monomer leaching into the aqueous continuous phase. Thus, the microfluidic platform and the unconventional setup, in which oil droplets containing both hydrophilic and hydrophobic components were polymerized in situ, allowed us to fabricate, for the first time, amphiphilic microgels based on sensitive cationic groups. The resulted microgel had an average diameter at around $18 \mu \mathrm{m}(\mathrm{CV} 7 \%)$.

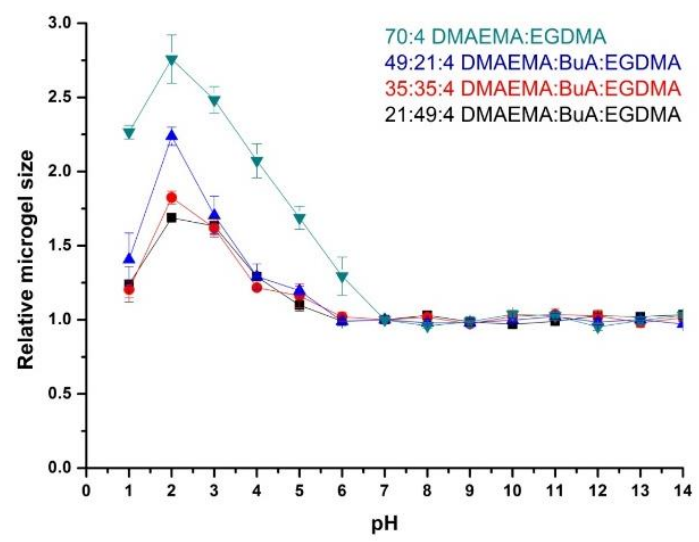

Figure 4 Swelling ratios of the DMAEMA-BuAEGDMA microgels and the DMAEMA-EGDMA microgel at different $\mathrm{pH}$ values. Microgels formed with DMAEMA:BuA:EGDMA molar ratios of 70:0:4, 49:21:4, 35:35:4 and 21:49:4 are represented as green triangles, blue triangles, red circles and black squares, respectively.

Three types of amphiphilic DMAEMA-BuAEDGMA microgels of differing compositions, in addition to a DMAEMA-EGDMA homopolymer microgel, were successfully fabricated. In all cases, the cross-linking density was kept the same (monomer:EGDMA 70:4), as in our previous studies, while the hydrophilic DMAEMA:hydrophobic BuA monomer molar ratio was varied between 41:29, 35:35, 21:49, and 70:0, thus altering the hydrophobic content.

The $\mathrm{pH}$ responsiveness, in terms of swelling and shrinking, of the microgels was studied by measuring the size of the microgels at different $\mathrm{pH}$ values, achieved by suspending the microgels in solutions prepared using $\mathrm{NaOH}$ and $\mathrm{HCl}$. As can be observed in Figure 4, the size of the microgels increased with decreasing $\mathrm{pH}$. This behaviour was expected since the DMAEMA units at low $\mathrm{pH}$ become ionised due to the 
presence of tertiary amine groups, in turn making the microgels more hydrophilic and allowing more water to enter the microgel, causing it to swell. Furthermore, the cationic charges of the DMAEMA groups repel each other and thus force the polymer chains to extend more within the microgel, also causing the microgel to swell. This has been observed previously for DMAEMA containing microgels ${ }^{53,67}$ and macrogels. ${ }^{68-70}$ Note that the size of the microgels at very acidic $\mathrm{pH}$ was reduced as a result of the increased ionic strength due to the greater $\mathrm{HCl}$ concentration, as has been observed in DMAEMA containing macrogels. ${ }^{68-70}$

At low $\mathrm{pH}$ values, it was also clear that the size of the amphiphilic microgels was influenced by the hydrophobic/ hydrophilic ratio. In particular, as the hydrophobic ratio decreased (i.e. greater DMAEMA:BuA ratio) the size of the microgels increased, as had been similarly observed in anionic unit containing microgels at higher pHs. ${ }^{50,51}$ At high $\mathrm{pH}$ values the DMAEMA units are less hydrophilic so since the BuA units are also hydrophobic the whole chains are more in a collapsed/shrunk state, and thus the DMAEMA:BuA ratio does not affect the size at $\mathrm{pH}>7$.

It should be mentioned that all of the $\mathrm{BuA}$ containing microgels appeared to be more stable than the DMAEMA homopolymer microgels (i.e. 70:4 DMAEMA:EGDMA) which, in some cases, swelled so much in acidic $\mathrm{pH}$ that they broke into smaller pieces (see video, ESI), an occurrence that has been observed in macrogels. ${ }^{71}$ Thus, this indicates that imposing an amphiphilic nature onto the microgels also improves their mechanical stability.

\section{$\mathbf{p K}_{\mathbf{a}} \mathrm{s}$ of Amphiphilic Microgels}

The hydrophobic content of the microgels also influenced their $\mathrm{p} K_{\mathrm{a}} \mathrm{s}$, as determined by potentiometric titration. Specifically, when the hydrophobic BuA content was varied from 70 , to 50 , to 30 and to $0 \%$ molar ratio $\left((\mathrm{BuA} /(\mathrm{BuA}+\mathrm{DMAEMA}) \times 100 \%)\right.$, the $\mathrm{p} K_{\mathrm{a}}$ values increased from 5.3 to $5.5,5.6$ and 6.0, respectively. Thus, the effective $\mathrm{p} K_{\mathrm{a}}$ values increased as the DMAEMA content of the amphiphilic microgels increased, or equivalently, as the BuA content decreased. In other words,
DMAEMA became a stronger base as the microgels became less hydrophobic. This is because a decrease in the BuA content caused an increase in the hydrophilicity and in the dielectric constant of the microgels, rendering ionisation easier and increasing the effective $\mathrm{p} K_{\mathrm{a}}{ }^{72}$ This has been similarly observed in amino (base) containing amphiphilic macrogels ${ }^{68,69,71,73}$ and amphiphilic polymers. ${ }^{74-77}$ The reverse trend has also been observed in anionic (meth)acrylic acid based amphiphilic macrogels ( $\mathrm{p} K_{a}$ increased with increasing hydrophobic content). ${ }^{71,73}$

\section{Dye Encapsulation and Release Studies}

Having established that the microfluidically fabricated microgels were $\mathrm{pH}$ responsive, their ability to encapsulate and release both hydrophilic and hydrophobic moieties was examined. The intent was two-fold: (i) to prove the amphiphilic nature of the cationic DMAEMA microgels, and (ii) to determine their potential as delivery vehicles for drugs of varying hydrophobicity/hydrophilicity, similar to macrogels. ${ }^{68,78}$ A hydrophobic dye (Nile Red) and a hydrophilic dye (Eriglaucine) were employed as model drugs for these studies, with their release triggered by swelling of the microgels.

\section{Hydrophobic dye - Nile Red}

Nile Red (Figure 3a) was encapsulated into the polymerized microgels by diffusion in the swollen state. DMAEMA49- $\mathrm{Co}-\mathrm{BuA}_{21}-\mathrm{Co}$ EGDMA $_{4}$ microgel was first suspended and swelled in Nile Red dye solution at $\mathrm{pH} 3$ for encapsulation (Figure 5a). With the help of sonication, Nile Red diffused into the microgel in the swollen state, which was fully encapsulated inside as the microgel shrank back when the basic solution ( $\mathrm{pH}$ 14) was added (Figure 5b). Upon addition of an acidic solution ( $\mathrm{pH} 3$ ), the aminobased functional groups were ionised and built up internal electrostatic repulsion between ionised cationic groups. Therefore, the polymer chains extended and force the microgel to swell, while the hydrophobic Nile Red could no longer be retained inside the microgel and thus was released into the surrounding medium over the course of $45 \mathrm{~min}$. This was evidenced by a 
reduction in colour intensity of the microgels over this time (Figure $5 \mathrm{c}$ and $5 \mathrm{~d}$ ).

The dye release rate was further investigated for each of the four types of microgel, and the results are shown in Figure 6. Each of the microgels was able to encapsulate and release the dye, but there was a clear influence of the hydrophobic content on the release rate. In particular, by increasing the hydrophobic monomer $(\mathrm{BuA})$ molar ratio content from $0 \%$ (70:0:4 DMAEMA:Bu:EGDMA) to $70 \%$ $(21: 49: 4)$, the rate of release of the hydrophobic dye decreased. This was expected since the hydrophobic components of the microgel retained the hydrophobic dye in the polymer structure to a greater extent, as a result of hydrophobic interactions. Also, due to the increased hydrophobic content, the microgels swelled less which likely slowed the diffusion of the dye through the microgels and consequently reduced the release rate.
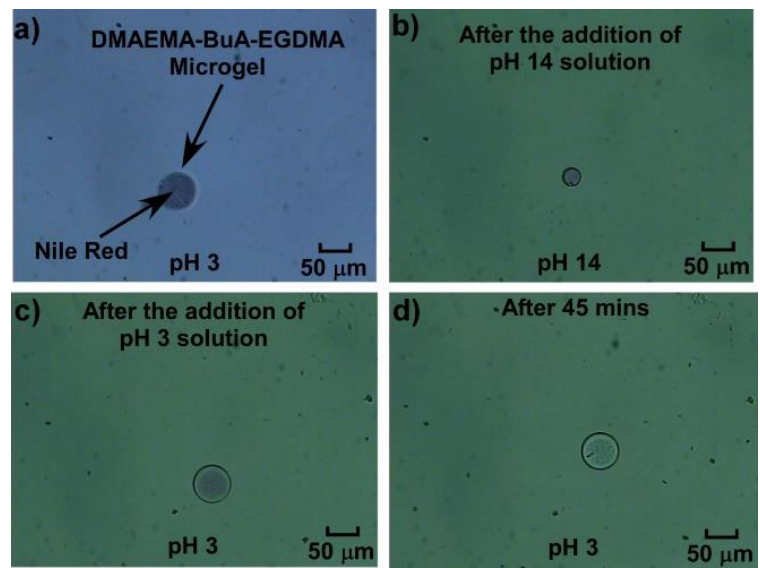

Figure 5 (a) DMAEMA $_{49}-\mathrm{Co}-\mathrm{BuA}_{21}-\mathrm{Co}$ EGDMA $_{4}$ microgel with hydrophobic Nile Red encapsulated inside at $\mathrm{pH}$ 3. (b) The same microgel immediately after addition of a $\mathrm{pH} 14$ solution, in which it formed a contracted state that held the Nile Red. (c) The same microgel immediately and (d) 45 minutes after the addition of a $\mathrm{pH} 3$ solution, demonstrating the release of Nile Red from the swollen microgel over time.

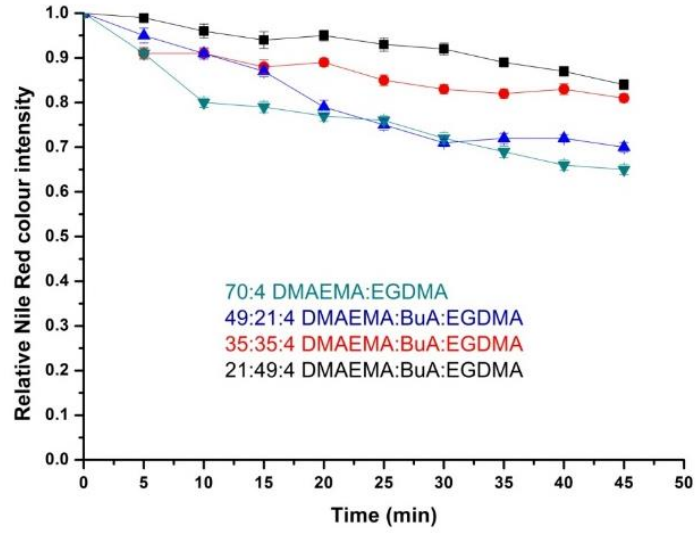

Figure 6 Hydrophobic drug release in acidic $(\mathrm{pH}$ 3 ) conditions. The plots show the relative Nile Red colour intensity versus time for four types of DMAEMA-BuA-EGDMA microgels with different DMAEMA:BuA molar ratios. Green triangles, blue triangles, red circles and black squares represent the microgels with 70:0:4, 49:21:4, 35:35:4 and 21:49:4 DMAEMA: BuA:EGDMA molar ratios, respectively.

\section{Hydrophilic dye - Eriglaucine}

The encapsulation and release of the hydrophilic dye, Eriglaucine (Figure 3b), was also investigated. Figure 7a shows the suspension of a DMAEMA49-co-BuA 21 -co-EGDMA 4 microgel in Erioglaucine dye solution in $\mathrm{pH} 3$ acidic solution, with sonication employed to enable diffusion of the dye into the microgel. Following this, $\mathrm{pH} 14$ basic solution was added in order to shrink the microgel to trap the dye (Figure 7b). When the $\mathrm{pH}$ was then decreased back to 3 and the microgel swelled, as described in previous sections, the blue colour of the microgel faded with time due to the gradual release of the hydrophilic Erioglaucine. 

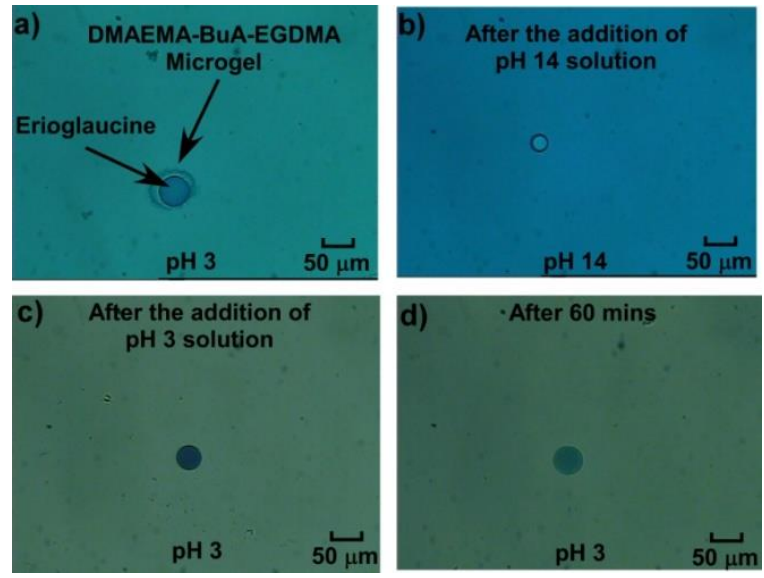

Figure 7 (a) DMAEMA $_{49}-\mathrm{CO}-\mathrm{BuA}_{21}-\mathrm{Co}$ $\mathrm{EGDMA}_{4}$ microgel with hydrophobic Erioglaucine disodium salt encapsulated at $\mathrm{pH} 3$. (b) The same microgel immediately after addition of $\mathrm{pH} 14$ solution, in which the microgel contracted and trapped the dye. (c) The microgel immediately following the addition of $\mathrm{pH} 3$ solution and (d) 60 minutes later.

The release of the hydrophilic dye was further investigated over time for all four types of DMAEMA-BuA-EGDMA microgels in acidic (pH 3) solution (Figure 8). The main observation made from the acquired data was that by decreasing the hydrophobic $(\mathrm{BuA})$ content, the hydrophilic dye release rate was increased, as was expected and observed for the hydrophobic dye as well as in our previous study on anionic amphiphilic microgels. ${ }^{50}$ This was due to the small degree of swelling when increasing the hydrophobic content that meant the pore/mesh size was smaller, thus preventing the dye from diffusing as easily through the microgel.

When comparing the hydrophobic Nile Red release to that of hydrophilic but charged Eriglaucine dye from the amphiphilic microgels, the latter demonstrated slower release rate for all four microgels at acidic pH. For example, it took around $45 \mathrm{~min}$ for the release of hydrophobic Nile Red from DMAEMA49-Co-BuMA ${ }_{21}-\mathrm{CO}$ $\mathrm{EGDMA}_{4}$ microgels at acidic $\mathrm{pH}$ to reach $70 \%$ of the relative colour intensity (compared to the initial intensity), while this level was reached in 60 min during hydrophilic Eriglaucine release. This can be attributed to two factors: (i) the existence of ethanol in the Nile Red dye mixture enhancing the release of hydrophobic dye and (ii) the attractive electrostatic interactions between the cationic microgels and the anionic Eriglaucine dye that would decelerate the release rate of dye from the microgel.

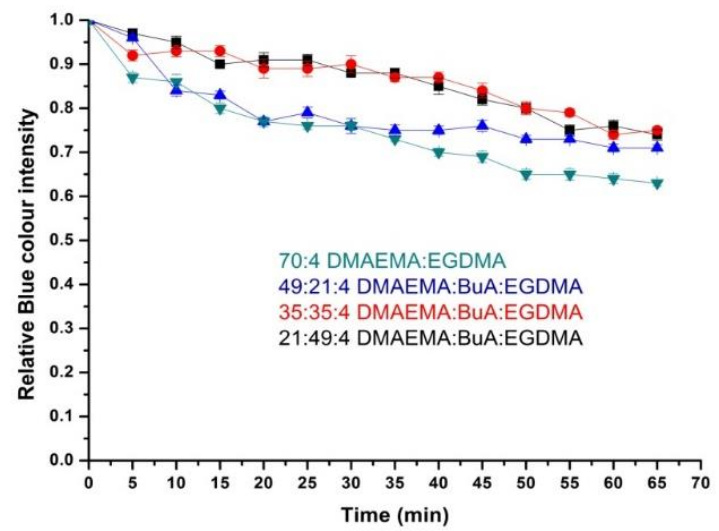

Figure 8 Hydrophilic dye release in acidic conditions $(\sim \mathrm{pH} 3)$. The plots show the relative Erioglaucine colour intensity versus time for each variation of the DMAEMA-BuA-EGDMA microgels. Green triangles, blue triangles, red circles and black squares represent the microgels with 70:0:4, 49:21:4, 35:35:4 and 21:49:4 DMAEMA:BuA:EGDMA molar ratios, respectively.

In summary, it was demonstrated that the novel cationic microgels were able to encapsulate and deliver both hydrophilic and hydrophobic dyes due to their amphiphilic nature. The tailorability of the release rate of both dyes by adjusting the hydrophobic content of the amphiphilic microgels was also demonstrated, which shows great promise for their use in future drug delivery applications.

\section{CONCLUSIONS}

Amphiphilic microgels based on hydrophilic, ionisable cationic DMAEMA monomer, hydrophobic non-ionic BuA monomer, and hydrophobic EGDMA cross-linker were successfully fabricated using a lab-on-a-chip platform via in situ generation and direct photopolymerization of spherical precursor droplets in an expanded serpentine channel. The use of the microfluidic device enabled the easy tailoring of the DMAEMA:BuA ratio and thus the amphiphilicity of the microgels. The microgels were $\mathrm{pH}$ responsive: by 
decreasing the $\mathrm{pH}$ of the surrounding environment the microgel swelled due to protonation of the DMAEMA units and the resultant electrostatic repulsion between the polymer chains inside the microgels. The extent of the swelling was controlled by varying the hydrophobic:hydrophilic ratio, with the size of the microgels decreasing with increasing hydrophobic $\mathrm{BuA}$ monomer content. Finally, the cationic amphiphilic microgels demonstrated the ability to deliver both hydrophobic and hydrophilic moieties. The release was influenced by both the hydrophobic content of the microgels and electrostatic interactions. Specifically, by increasing the hydrophilic content of the microgels the release rate was accelerated, while the anionic dye was released at slower rates than the non-ionic dye due to attractive electrostatic interactions with the microgels. These properties show great promise for the continuous, automated, in situ preparation of cationic amphiphilic microgels that could be applied to the delivery of both hydrophilic and hydrophobic drugs.

\section{ACKNOWLEDGEMENTS}

University of Hull is thanked for funding $\mathrm{Mr}$ Lu's PhD studentship. Dr Stephen Clark is thanked for fabrication of the microfluidic devices.

\section{REFERENCES AND NOTES}

1. V. Alakhov, G. Pietrzynski, K. Patel, A. Kabanov, L. Bromberg, T. A. Hatton. J. Pharm. Pharmacol. 2004, 56, 1233-1241.

2. Y. Jiang, J. Chen, C. Deng, E. J. Suuronen, Z. Zhong. Biomaterials 2014, 35, 4969-4985.

3. Y. Gao, A. Ahiabu, M. J. Serpe. ACS Appl. Mater. Interfaces 2014, 6, 13749-13756.

4. L. Bromberg, M. Temchenko, T. A. Hatton. Langmuir 2002, 18, 4944-4952.

5. J. P. K. Tan, K. C. Tam. J. Controlled Release 2007, 118, 87-94.

6. W. C. Lee, Y. C. Li, I. M. Chu. Macromol. Biosci. 2006, 6, 846-854.

7. L. Li, C. Cheng, M. P. Schürings, X. Zhu, A. Pich. Polymer 2012, 53, 3117-3123.
8. M. Dadsetan, K. E. Taylor, C. Yong, Ž. Bajzer, L. Lu, M. J. Yaszemski. Acta Biomaterialia 2013, 9, 5438-5446.

9. H. Bysell, R. Månsson, P. Hansson, M. Malmsten. Adv. Drug Delivery Rev. 2011, 63, 1172-1185.

10. T. Trongsatitkul, B. M. Budhlall. Polym. Chem. 2013, 4, 1502-1516.

11. T. K. Georgiou, M. Vamvakaki, L. A. Phylactou, C. S. Patricklos. Biomacromolecules 2005, 6, 2990-2997.

12. T. K. Georgiou, M. Vamvakaki, C. S. Patrickios, E. N. Yamasaki, L. A. Phylactou. Biomacromolecules 2004, 5, 2221-2229.

13. T. K. Georgiou, L. A. Phylactou, C. S. Patrickios. Biomacromolecules 2006, 7, 35053512.

14. N. J. Zuidam, G. Posthuma, E. T. J. De Vries, D. J. A. Crommelin, W. E. Hennink, G. Storm. J. Drug Targeting 2000, 8, 51-66.

15. F. J. Verbaan, P. K. Klouwenberg, J. H. Van Steenis, C. J. Snel, O. Boerman, W. E. Hennink, G. Storm. Int. J. Pharm. 2005, 304, 185-192.

16. P. Van De Wetering, E. E. Moret, N. M. E. Schuurmans-Nieuwenbroek, M. J. Van Steenbergen, W. E. Hennink. Bioconjugate Chem. 1999, 10, 589-597.

17. C. Siltanen, M. Yaghoobi, A. Haque, J. You, J. Lowen, M. Soleimani, A. Revzin. Acta Biomaterialia 2016, 34, 125-132.

18. W. Zhou, J. M. Stukel, H. L. Cebull, R. K. Willits. Macromol. Biosci. 2016, 16, 535-544.

19. A. Paul, V. Manoharan, D. Krafft, A. Assmann, J. A. Uquillas, S. R. Shin, A. Hasan, M. A. Hussain, A. Memic, A. K. Gaharwar, A. Khademhosseini. J. Mater. Chem. B 2016, 4, 3544-3554.

20. M. R. Islam, A. Ahiabu, X. Li, M. J. Serpe. Sensors 2014, 14, 8984-8995.

21. X. Zhang, C. Gao, S. Lü, H. Duan, N. Jing, D. Dong, C. Shi, M. Liu. J. Mater. Chem. B 2014, 2, 5452-5460.

22. Y. Chen, Y. Bai, S. Chen, J. Jup, Y. Li, T. Wang, Q. Wang. ACS Appl. Mater. Interfaces 2014, 6, 13334-13338.

23. D. Parasuraman, M. J. Serpe. ACS Appl. Mater. Interfaces 2011, 3, 4714-4721.

24. D. Parasuraman, M. J. Serpe. ACS Appl. Mater. Interfaces 2011, 3, 2732-2737.

25. C. D. Sorrell, M. C. D. Carter, M. J. Serpe. ACS Appl. Mater. Interfaces 2011, 3, 1140-1147. 
26. A. I. Triftaridou, F. Tournilhac, L. Leibler, S. Auguste, J. M. Pernot. Polym. Chem. 2013, 4, 2125-2131.

27. E. M. Rosenbauer, A. I. Triftaridou, S. Karpati, F. Tournilhac, L. Leibler, S. Auguste, J. M. Pernot. Polym. Chem. 2012, 3, 2178-2185.

28. P. Glampedaki, J. Krägel, G. Petzold, V. Dutschk, R. Miller, M. M. C. G. Warmoeskerken. Colloid Surf. A-Physicochem. Eng. Asp. 2012, 413, 334-341.

29. P. Glampedaki, A. Calvimontes, V. Dutschk, M. M. C. G. Warmoeskerken. J. Mater. Sci. 2012, 47, 2078-2087.

30. A. Kulkarni, A. Tourrette, M. M. C. G. Warmoeskerken, D. Jocic. Carbohydr. Polym. 2010, 82, 1306-1314.

31. H. M. Shewan, J. R. Stokes. J. Food Eng. 2013, 119, 781-792.

32. C. S. Patrickios, T. K. Georgiou. Curr. Opin. Colloid Interface Sci. 2003, 8, 76-85.

33. Anon. Macromolecules 1990, 23, 5158-5160.

34. L. Bromberg, M. Temchenko, G. D. Moeser, T. A. Hatton. Langmuir 2004, 20, 5683-5692.

35. X. Wang, X. Hou, Y. Wu, S. You. J. Appl. Polym. Sci. 2009, 114, 4042-4050.

36. H. Li, X. Cui, S. Shen, D. Hu. J. Appl. Polym. Sci. 2011, 122, 509-516.

37. H. Li, P. Zhang, L. Zhang, T. Zhou, D. Hu. J. Mater. Chem. 2009, 19, 4575-4586.

38. T. Zhou, H. Li, G. Liu, L. Zhang, D. Yao, D. Hu. J. Appl. Polym. Sci. 2009, 114, 4000-4010.

39. M. D. Tarn, N. Pamme, In Elsevier Reference Module in Chemistry, Molecular Sciences and Chemical Engineering; Reedijk, J., Ed.; Elsevier: Waltham, MA, 2013.

40. H. Song, D. L. Chen, R. F. Ismagilov. Angew. Chem. - Int. Ed. 2006, 45, 7336-7356.

41. S.-Y. Teh, R. Lin, L.-H. Hung, A. P. Lee. Lab Chip 2008, 8, 198-220.

42. E. Tumarkin, E. Kumacheva. Chem. Soc. Rev. 2009, 38, 2161-2168.

43. S. Seiffert, D. A. Weitz. Polymer 2010, 51, 5883-5889.

44. W. J. Duncanson, T. Lin, A. R. Abate, S. Seiffert, R. K. Shah, D. A. Weitz. Lab on a Chip - Miniaturisation for Chemistry and Biology 2012, 12, 2135-2145.

45. S. Seiffert. Angew. Chem. - Int. Ed. 2013, 52, 11462-11468.

46. M. T. Gokmen, F. E. Du Prez. Prog. Polym. Sci. 2012, 37, 365-405.
47. R. A. Prasath, M. T. Gokmen, P. Espeel, F. E. Du Prez. Polym. Chem. 2010, 1, 685-692.

48. M. T. Gokmen, W. Van Camp, P. J. Colver, S. A. F. Bon, F. E. Du Prez. Macromolecules 2009, 42, 9289-9294.

49. P. Van De Watering, N. J. Zuidam, M. J. Van Steenbergen, O. A. G. J. Van Der Houwen, W. J. M. Underberg, W. E. Hennink. Macromolecules 1998, 31, 8063-8068.

50. B. Lu, M. D. Tarn, N. Pamme, T. K. Georgiou. Journal of Materials Chemistry B 2016.

51. B. Lu, M. D. Tarn, N. Pamme, T. K. Georgiou. J. Mater. Chem. B 2015, 3, 4524-4529. 52. G. Liu, D. Wang, F. Zhou, W. Liu. Small 2015, 11, 2807-2816.

53. Y. Tang, T. Wu, B. Hu, Q. Yang, L. Liu, B. Yu, Y. Ding, S. Ye. Mater. Chem. Phys. 2015, 149, 460-466.

54. X. Zhang, S. Lü, C. Gao, C. Chen, X. Zhang, M. Liu. Nanoscale 2013, 5, 6498-6506.

55. C. Gao, M. Liu, J. Chen, C. Chen. Polym. Adv. Technol. 2012, 23, 389-397.

56. C. Monteux, C. Marliére, P. Paris, N. Pantoustier, N. Sanson, P. Perrin. Langmuir 2010, 26, 13839-13846.

57. B. G. De Geest, S. De Koker, J. Demeester, S. C. De Smedt, W. E. Hennink. J. Controlled Release 2009, 135, 268-273.

58. R. K. Shah, H. C. Shum, A. C. Rowat, D. Lee, J. J. Agresti, A. S. Utada, L.-Y. Chu, J.-W. Kim, A. Fernandez-Nieves, C. J. Martinez, D. A. Weitz. Materials Today 2008, 11, 18-27.

59. A. Emileh, E. Vasheghani-Farahani, M. Imani. Macromol. Symp. 2007, 255, 1-7.

60. F. Zhou, S. Li, C. D. Vo, J. J. Yuan, S. Chai, Q. Gao, S. P. Armes, C. Lu, S. Cheng. Langmuir 2007, 23, 9737-9744.

61. L. Hu, L. Y. Chu, M. Yang, H. D. Wang, C. Hui Niu. J. Colloid Interface Sci. 2007, 311, 110117.

62. D. Dupin, S. P. Armes, C. Connan, P. Reeve, S. M. Baxter. Langmuir 2007, 23, 6903-6910. 63. J. I. Amalvy, E. J. Wanless, Y. Li, V. Michailidou, S. P. Armes, Y. Duccini. Langmuir 2004, 20, 8992-8999.

64. L. Zha, J. Hu, C. Wang, S. Fu, A. Elaissari, Y. Zhang. Colloid Polym. Sci. 2002, 280, 1-6.

65. T. McCreedy. Anal. Chim. Acta 2001, 427, $39-43$. 
66. A. Pal, S. Chaudhary. Fluid Phase Equilibria 2014, 372, 100-104.

67. R. B. Karabacak. J. Appl. Polym. Sci. 2015. 68. N. Ghasdian, E. Church, A. P. Cottam, K. Hornsby, M. Y. Leung, T. K. Georgiou. RSC Advances 2013, 3, 19070-19080.

69. T. K. Georgiou, C. S. Patrickios, P. W. Groh, B. Iván. Macromolecules 2007, 40, 2335-2343.

70. D. S. Achilleos, T. K. Georgiou, C. S. Patrickios. Biomacromolecules 2006, 7, 33963405.

71. M. Vamvakaki, C. S. Patrickios. Soft Matter 2008, 4, 268.

72. O. E. Philippova, D. Hourdet, R. Audebert, A. R. Khokhlov. Macromolecules 1997, 30, 82788285.

73. A. I. Triftaridou, S. C. Hadjiyannakou, M. Vamvakaki, C. S. Patrickios. Macromolecules 2002, 35, 2506-2513.
74. M. A. Ward, T. K. Georgiou. J. Polym. Sci., Part A: Polym. Chem. 2010, 48, 775-783.

75. M. A. Ward, T. K. Georgiou. Soft Matter 2012, 8, 2737-2745.

76. M. A. Ward, T. K. Georgiou. Polym. Chem. 2013, 4, 1893-1902.

77. A. P. Constantinou, T. K. Georgiou. Polym. Chem. 2016, 7, 2045-2056.

78. P. Papaphilippou, M. Christodoulou, O. M. Marinica, A. Taculescu, L. Vekas, K. Chrissafis, T. Krasia-Christoforou. ACS Appl. Mater. Interfaces 2012, 4, 2139-2147. 


\section{GRAPHICAL ABSTRACT}

Bingyuan Lu, Mark D. Tarn, Nicole Pamme, Theoni K. Georgiou

Fabrication of Tailorable pH Responsive Cationic Amphiphilic Microgels on a Microfluidic Device for Drug Release

Novel cationic amphiphilic microgels with hydrophobic and hydrophilic monomer units on the polymer chains were fabricated with an on chip polymerisation methodology using a novel chip design.

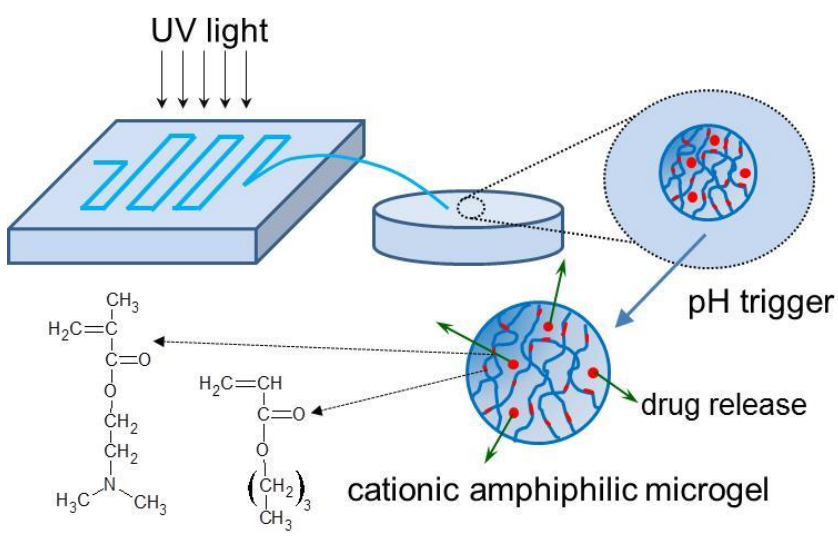

